\title{
APPROXIMATION OF THE EQUILIBRIUM DISTRIBUTION BY DISTRIBUTIONS OF EQUAL POINT CHARGES WITH MINIMAL ENERGY
}

\author{
J. KOREVAAR AND M. A. MONTERIE
}

Abstract. Let $\omega$ denote the classical equilibrium distribution (of total charge 1 ) on a convex or $C^{1, \alpha}$-smooth conductor $K$ in $\mathbb{R}^{q}$ with nonempty interior. Also, let $\omega_{N}$ be any $N$ th order "Fekete equilibrium distribution" on $K$, defined by $N$ point charges $1 / N$ at $N$ th order "Fekete points". (By definition such a distribution minimizes the energy for $N$-tuples of point charges $1 / N$ on $K$.) We measure the approximation to $\omega$ by $\omega_{N}$ for $N \rightarrow \infty$ by estimating the differences in potentials and fields,

$$
U^{\omega}-U^{\omega_{N}} \text { and } \mathcal{E}^{\omega}-\mathcal{E}^{\omega_{N}},
$$

both inside and outside the conductor $K$. For dimension $q \geq 3$ we obtain uniform estimates $\mathcal{O}\left(1 / N^{1 /(q-1)}\right)$ at distance $\geq \varepsilon>0$ from the outer boundary $\Sigma$ of $K$. Observe that $\mathcal{E}^{\omega}=0$ throughout the interior $\Omega$ of $\Sigma$ (Faraday cage phenomenon of electrostatics), hence $\mathcal{E}^{\omega_{N}}=\mathcal{O}\left(1 / N^{1 /(q-1)}\right)$ on the compact subsets of $\Omega$.

For the exterior $\Omega^{\infty}$ of $\Sigma$ the precise results are obtained by comparison of potentials and energies. Admissible sets $K$ have to be regular relative to capacity and their boundaries must allow good Harnack inequalities. For the passage to interior estimates we develop additional machinery, including integral representations for potentials of measures on Lipschitz boundaries $\Sigma$ and bounds on normal derivatives of interior and exterior Green functions.

Earlier, one of us had considered approximations to the equilibrium distribution by arbitrary distributions $\mu_{N}$ of equal point charges on $\Sigma$. In that context there is an important open problem for the sphere which is discussed at the end of the paper.

\section{INTRODUCTION AND RESULTS}

1.1. The problem. Let $K$, "the conductor", be a compact set in $\mathbb{R}^{q}$ of positive capacity. The unbounded component of the complement $K^{c}$, to which one adds a single point at $\infty$, is denoted by $K^{\infty}$. We write $\Sigma$ for the outer boundary of $K$, $\Sigma=\partial_{0} K=\partial K^{\infty}$.

One considers all probability measures $\mu$ on $K$. For such "distributions $\mu$ of positive charge" on $K$, of total charge 1 , the electrostatic potential, the electrostatic

Received by the editors April 1, 1996.

1991 Mathematics Subject Classification. Primary 31B15; Secondary 31B05, 31B10, 31B25.

Key words and phrases. Capacity, capacity-regular sets, electrostatic fields, energies, equilibrium distributions, Fekete points, Green functions and their gradients, harmonic functions, harmonic measure, Harnack-type inequalities, integral representations, Kelvin transform, level surfaces, Lipschitz domains, potentials. 
field and the energy are defined by

$$
\begin{gathered}
U^{\mu}(x)=\int_{K} \Phi(|x-y|) d \mu(y), \quad \mathcal{E}^{\mu}(x)=-\operatorname{grad} U^{\mu}(x), \\
I(\mu)=\int_{K \times K} \Phi(|x-y|) d \mu(x) d \mu(y),
\end{gathered}
$$

where $\Phi=\Phi_{q}$,

$$
\Phi_{q}(r)=1 / r^{q-2} \quad \text { if } q \geq 3, \quad \Phi_{2}(r)=\log (1 / r) .
$$

Among the probability measures $\mu$ on $K$ there is a unique equilibrium distribution $\omega$ which minimizes the energy. The minimal energy $I(\omega)$ gives the Robin constant $V$ for $K$. One has $\operatorname{cap} K=\Phi^{-1}(V), \operatorname{supp} \omega \subset \Sigma, U^{\omega} \leq V$ throughout $\mathbb{R}^{q}, U^{\omega}=V$ on $K$ outside an exceptional subset of $\Sigma$ of (outer) capacity zero, see Frostman [7]. If $K^{\infty}$ is regular for the Dirichlet problem, $U^{\omega}=V$ everywhere on $K$ and inside $\Sigma$, cf. Wermer [33].

Fixing $N$ (always $\geq 2$ ) for a moment, we also consider all special discrete measures $\mu_{N}=\mu_{N}\left(x_{1}, \ldots, x_{N}\right)$ given by charges $1 / N$ at (distinct) points $x_{1}, \ldots, x_{N} \in$ $K$. The corresponding electrostatic potential and so-called discrete energy are given by

$$
U^{\mu_{N}}(x)=\frac{1}{N} \sum_{k=1}^{N} \Phi\left(\left|x-x_{k}\right|\right), \quad I^{*}\left(\mu_{N}\right) \stackrel{\text { def }}{=} \frac{1}{N^{2}} \sum_{j, k=1, j \neq k}^{N} \Phi\left(\left|x_{j}-x_{k}\right|\right) .
$$

(The ordinary energy $I\left(\mu_{N}\right)$ is infinite.) Any measure $\mu_{N}$ for which the discrete energy $I^{*}\left(\mu_{N}\right)$ is minimal will be called an $N$ th order "Fekete measure" $\omega_{N}$ on $K$. Its support is an $N$-tuple $\left\{f_{N 1}, \ldots, f_{N N}\right\}$ of $N$ th order "Fekete points" which belong to the outer boundary $\Sigma$ of $K$. Such points were introduced by Fekete [6] for dimension $q=2$.

One can show that $I^{*}\left(\omega_{N}\right) \rightarrow I(\omega)$ as $N \rightarrow \infty$ (cf. Remark 3.11), from which it follows that $\omega_{N} \rightarrow \omega$ in weak ${ }^{*}$ sense (cf. Frostman [7]). Thus $\omega_{N}(E) \rightarrow \omega(E)$ for well-behaved subsets $E \subset \Sigma$ as $N \rightarrow \infty$, and

$$
U^{\omega_{N}}(x) \rightarrow U^{\omega}(x), \quad \mathcal{E}^{\omega_{N}}(x) \rightarrow \mathcal{E}^{\omega}(x), \quad \forall x \in \mathbb{R}^{q}-\Sigma .
$$

Question. How small are the differences

$$
\omega(E)-\omega_{N}(E), \quad U^{\omega}(x)-U^{\omega_{N}}(x), \quad \mathcal{E}^{\omega}(x)-\mathcal{E}^{\omega_{N}}(x)
$$

for large values of $N$ ?

Observe that $\mathcal{E}^{\omega}=0$ everywhere inside $\Sigma$, an idealized mathematical formulation of the classical Faraday-cage phenomenon of electrostatics. One would like to know in particular how small $\mathcal{E}^{\omega_{N}}$ will be inside $\Sigma$.

For a conductor $K$ given by an analytic or $C^{3, \alpha}$ Jordan curve $\Gamma$ in the plane, Pommerenke [31] and Korevaar and Kortram [18, 23] have obtained close approximations to the Fekete points. For very smooth $\Gamma$, the approximations show that $\omega(E)-\omega_{N}(E)=\mathcal{O}(1 / N)$, uniformly for the subarcs $E \subset \Gamma$. Via a Stieltjes integral for the potential difference $U^{\omega}-U^{\omega_{N}}$ this estimate implies that $\mathcal{E}^{\omega}-\mathcal{E}^{\omega_{N}}$ is at most of order $1 / N$ at distance $\geq \epsilon>0$ from the curve, see Korevaar and coauthors Geveci and Kortram [18, 22, 23]. These papers also show that the order $\mathcal{O}(1 / N)$ is sharp except when $\Gamma$ is a circle.

For less smooth conductors in the plane (such as a square) and for conductors in higher dimensions, the known results on the distribution of Fekete points (cf. 
Sjögren [32], Dahlberg [3]) do not suffice to prove the desired estimates for the differences in potentials and fields. Thus an entirely new method of proof is required (see Section 1.3).

1.2. Principal results. Throughout this paper, $\Omega$ denotes a bounded domain in $\mathbb{R}^{q} \cup\{\infty\}$ which is the interior of its outer boundary. Thus $\Omega$ and the complement $\Omega^{\infty}$ of the closure $\bar{\Omega}$ have the same boundary $\Sigma$. For the time being we suppose that the "conductor" $K$ is the closure of $\Omega$ or its boundary $\Sigma$. Later we will allow more general compact sets $K$, at least for the estimates on the unbounded domain $K^{\infty}$.

Theorem 1.1. Let the bounded domain $\Omega \subset \mathbb{R}^{q}(q \geq 3)$ be convex or have $C^{1, \alpha}$ boundary $\Sigma$ (with $\alpha>0)$. Then for $N \geq 2$ and $x \in \Omega$ or $x \in \Omega^{\infty}$,

$$
-c_{1} \min \left(1, \frac{1}{d_{x}^{q-2}}\right) \frac{1}{N^{1 /(q-1)}} \leq U^{\omega_{N}}(x)-U^{\omega}(x) \leq c_{2} \frac{1+d_{x}}{d_{x}^{q-1}} \frac{1}{N^{1 /(q-1)}},
$$

where $d_{x}=d(x, \Sigma)$ and the constants $c_{j}$ depend only on $\Omega$.

Once Theorem 1.1 has been established, one may use the Poisson integral to obtain an upper bound for the difference of the electrostatic fields. The result is

Theorem 1.2. Under the hypotheses of Theorem 1.1, there is a constant $c_{3}$ depending only on $\Omega$ such that for $N \geq 2$ and all $x \notin \Sigma$,

$$
\left|\mathcal{E}^{\omega}(x)-\mathcal{E}^{\omega_{N}}(x)\right| \leq c_{3} \frac{1+d_{x}}{d_{x}^{q}} \frac{1}{N^{1 /(q-1)}} .
$$

Remarks 1.3. Sjögren's results [32] for Fekete points on $C^{1, \alpha}$ surfaces imply estimates in (1.4) and (1.5) of order $1 / N^{1 /(2 q-2)}$ when $q \geq 3$.

For convex or $C^{1, \alpha}$-smooth conductors $K$ in dimension $q=2$, our method will give order $\mathcal{O}((\log N) / N)$ which is close to the known optimal order $\mathcal{O}(1 / N)$ for very smooth conductors in the plane (different from circles). Thus it is plausible that the orders in the theorems above are best possible relative to $N$ (except perhaps for spheres).

Pommerenke's paper [30] implies an order estimate $\mathcal{O}((\log N) / N)$ for $q=2$ under weaker conditions on $K$, but only for the unbounded domain $K^{\infty}$.

1.3. Method of proof. Since the proof of Theorem 1.1 is quite long we present a detailed outline here. Precise results will be given in subsequent sections.

Step 1. In order to manufacture a positive potential, we start with a lower bound for the difference $U^{\omega_{N}}-U^{\omega}$ on $K$ or $\Sigma$. Here $U^{\omega} \equiv V=V(K)$, the Robin constant for $K$. In Chapter 3, comparison of energies in the spirit of Pólya and Szegö [29] and a useful observation by Sjögren [32] lead to a lower estimate

$$
U^{\omega_{N}}-U^{\omega} \geq-\delta\left(\omega_{N}\right) \quad \text { on } \Sigma, \quad \text { with } 0<\delta\left(\omega_{N}\right) \leq \frac{c_{1}}{N^{1 /(q-1)}} .
$$

The result extends to all of $\mathbb{R}^{q}$ by the minimum principle for harmonic functions.

Step 2. We next form the auxiliary potential

$$
\begin{gathered}
T=U^{\rho_{N}} \stackrel{\text { def }}{=} U^{\omega_{N}}-U^{\omega}+\frac{1}{V} \delta\left(\omega_{N}\right) U^{\omega}, \\
\rho_{N} \stackrel{\text { def }}{=} \omega_{N}-\omega+\frac{1}{V} \delta\left(\omega_{N}\right) \omega, \quad \operatorname{supp} \rho_{N} \subset \Sigma .
\end{gathered}
$$

By (1.6), $T \geq 0$ on $\Sigma$, hence $T>0$ throughout $\mathbb{R}^{q}-\Sigma$. 
Step 3. If $\Sigma$ is the unit sphere $S=S(0,1)$ one has $U^{\omega_{N}}(0)=U^{\omega}(0)=1$ and $V=1$, so that $T(0)=\delta\left(\omega_{N}\right)$. One may then use the Poisson integral or Harnack's inequality to prove that $T$, and hence $U^{\omega_{N}}-U^{\omega}<T$, is $\leq \mathcal{O}\left(\delta\left(\omega_{N}\right)\right)$ at distance $\geq \varepsilon>0$ from $\Sigma$, cf. Korevaar's report [19] for $q=3$.

In the general case there is usually no finite center where one has information about the value of $T$. However, we do have information at infinity:

$$
L(T) \stackrel{\text { def }}{=} \lim _{x \rightarrow \infty}|x|^{q-2} T(x)=\frac{1}{V} \delta\left(\omega_{N}\right)
$$

since $\lim |x|^{q-2} U^{\mu}(x)=1$ for all probability measures $\mu$.

For $x$ in the exterior domain $\Omega^{\infty}$ one may now use a Harnack inequality around $\infty$ to conclude that for the positive harmonic function $T$,

$$
T(x) \leq c\left(d_{x}\right) L(T), \quad d_{x}=d(x, \Sigma) .
$$

To verify this one may apply Kelvin inversion with center $0 \in \Omega$; for finite domains there is a general Harnack inequality in Gilbarg and Trudinger [11]. For our domains one can estimate $c\left(d_{x}\right) \leq c^{\prime}\left(1+d_{x}\right) / d_{x}^{q-1}$, cf. Section 2.3. Combining (1.9) with (1.6) - (1.8) one obtains the upper bound in (1.4) for the exterior domain.

Step 4 . To go from the exterior to the interior we establish a general inequality

$$
T(x) \leq A(x, \Sigma) L(T), \quad x \in \Omega,
$$

for nonnegative potentials $T$ of signed measures on $\Sigma$. The proof of (1.10) uses integral representations for $T(x)$ and $L(T)$ in terms of the values of $T$ on $\Sigma$ (Chapter 4).

Convex or $C^{1, \alpha}$-smooth domains $\Omega$ are special cases of Lipschitz domains. One says that the (bounded) domain $\Omega$ in $\mathbb{R}^{q}$ is a Lipschitz domain, or that $\Sigma=\partial \Omega$ is a Lipschitz surface, if for every $x \in \Sigma$ there are a neighborhood $\mathcal{U}$ of $x$, a coordinate system $(\xi, \eta), \xi \in \mathbb{R}^{q-1}, \eta \in \mathbb{R}$, and a Lipschitz continuous function $\varphi$ such that $\Omega \cap \mathcal{U}=\{(\xi, \eta): \varphi(\xi)<\eta\} \cap \mathcal{U}$.

Using the same notation, $\Sigma$ (or $\Omega$ ) is said to be of class $C^{1, \alpha}(0<\alpha<1)$ if the function $\varphi$ is of class $C^{1}$ and its gradient is Hölder continuous with exponent $\alpha$.

For harmonic functions on a Lipschitz domain which are continuous up to the boundary $\Sigma$ there is an integral representation involving normal derivatives of Green functions, cf. Dahlberg's work [2] on harmonic measure. This representation will be extended to potentials $T$ of measures on $\Sigma$. For the exterior domain we obtain an integral representation for the limit $L(T)$ at $\infty$ involving the Green function with pole at $\infty$.

In order to get simple representations we use adjusted area measure $\sigma(y)=$ $\lambda(y) /(q-2) \lambda(S)$, where $\lambda$ is Lebesgue area measure on $\Sigma$ and $S=S^{q-1}$ is the unit sphere in $\mathbb{R}^{q}$. For the convex and $C^{1, \alpha}$ case considered in Theorem 1.1 we have useful inequalities for the "interior" normal derivatives of the Green functions. Taking $x \in \Omega$ we are thus able to estimate as follows:

$$
\begin{aligned}
T(x) & =\int_{\Sigma} T(y) \frac{\partial G}{\partial n_{y}}(y, x) d \sigma(y) \leq M(x) \int_{\Sigma} T(y) d \sigma(y) \\
& \leq \frac{M(x)}{m} \int_{\Sigma} T(y) \frac{\partial G}{\partial n_{y}}(y, \infty) d \sigma(y)=\frac{M(x)}{m} L(T) \leq \frac{c^{\prime \prime}}{d_{x}^{q-1}} L(T) .
\end{aligned}
$$

Indeed, $\left(\partial G / \partial n_{y}\right)(y, x)$ will be bounded above by a constant $M(x) \leq c / d_{x}^{q-1}$ for $x \in \Omega$ and $y \in \Sigma$, while $\left(\partial G / \partial n_{y}\right)(y, \infty)$ is bounded from below by a positive 
constant $m$ (Section 2.2). Combination of (1.11) with (1.6) - (1.8) gives the upper bound in (1.4) for the interior domain.

As a by-product of integral representations we obtain an expression for the classical equilibrium measure $\omega$ on Lipschitz boundaries in terms of the Green function with pole at $\infty$, see Section 4.4.

The authors are indebted to the referee and to N.J. Korevaar for useful observations.

\section{Auxiliary nOtions AND RESUlts}

2.1. Kelvin transformation. For the study of harmonic functions on unbounded domains such as $\Omega^{\infty}$ in $\mathbb{R}^{q}, q \geq 2$, it is convenient to use inversion or reflection with respect to the unit sphere:

$$
y=j(x) \stackrel{\text { def }}{=} \frac{x}{|x|^{2}}, \quad x \in \mathbb{R}^{q}-\{0\}, \quad j(0)=\infty, \quad j(\infty)=0 .
$$

Observe that $j^{-1}=j$. Except at 0 and $\infty$, inversion is angle-preserving and of class $C^{\infty}$. Thus if $\Sigma=\partial \Omega$ is a Lipschitz or $C^{1, \alpha}$ surface not passing through 0 , then the reflected surface $j(\Sigma)$ is also Lipschitz, or $C^{1, \alpha}$, respectively. For later use we record that the linear scaling factor at $x$ under inversion is equal to $1 /|x|^{2}$.

The Kelvin transform $v=k u=k(u)$ of a function $u$ on a domain $D$ in $\mathbb{R}^{q} \cup\{\infty\}$ is given by

$$
v(y)=(k u)(y)=\frac{1}{|y|^{q-2}} u \circ j(y), \quad y \in j(D) .
$$

It is well-known that a function $u$ is harmonic at (that is, in a neighborhood of) a point $x_{0} \neq 0$ if and only if $k u$ is harmonic at $j\left(x_{0}\right)$, cf. Kellogg [15] for $q=3$. A function $u$ is said to be harmonic at $\infty$ if and only if its Kelvin transform $k u$ is harmonic (or has a removable singularity) at 0 . Thus if $u$ is harmonic for $R<|x|<\infty$, it is harmonic at $\infty$ if and only if the limit

$$
L(u)=\lim _{x \rightarrow \infty}|x|^{q-2} u(x)=\lim _{y \rightarrow 0}(k u)(y)
$$

exists and is finite. In dimension $q \geq 3$ it will actually be sufficient if $\lim _{x \rightarrow \infty} u(x)=$ 0 . For $q \geq 3$ the potential $U^{\mu}$ of a (finite) measure $\mu$ with compact support is harmonic at $\infty$.

2.2. Green functions for interior and exterior. For unbounded domains such as our $\Omega^{\infty}$ in $\mathbb{R}^{q}$, the Green function $G(y, x)$ with pole at $x$ can be expressed in terms of the Green function $G^{\prime}$ for a bounded inverse domain. Taking $0 \in \Omega, q \geq 3$ and $x$ finite one has

$$
G(y, x)=\frac{1}{|y|^{q-2}|x|^{q-2}} G^{\prime}(j(y), j(x)), \quad y, x \in \Omega^{\infty},
$$

with obvious modification if $q=2$.

The important case of Green functions with pole at $\infty$ is different. We again take dimension $q \geq 3$; the case $q=2$ is well-known from complex analysis.

Definition 2.1. Let $K \subset \mathbb{R}^{q}, q \geq 3$, be compact and such that the unbounded component $K^{\infty}$ of its complement (including $\infty$ ) is regular for the Dirichlet problem. Then the Green function $G(y, \infty)$ for $K^{\infty}$ with pole at $\infty$ is the harmonic function on the finite part of $K^{\infty}$ with boundary values 0 on $\partial K^{\infty}$ and boundary value 1 at $\infty$. 
Observe that $G(y, \infty)=1-w(y)$, where $w$ is the harmonic function on $K^{\infty}$ with boundary function 1 on $\partial K^{\infty}$. There is a close relation between the Green function $G(y, \infty)$ and the equilibrium potential $U^{\omega}(y)$ : for compact $K$ as in Definition 2.1 and $V=V(K)$,

$$
G(y, \infty)=1-\frac{1}{V} U^{\omega}(y)=1-(\operatorname{cap} K)^{q-2} U^{\omega}(y), \quad y \in K^{\infty} .
$$

One may use relation (2.5) to define a generalized Green function $G(y, \infty)$ for $K^{\infty}$ whenever $K$ is a compact set of positive capacity. We observe for later use that

$$
\begin{gathered}
G(y, \infty)=1-\frac{1}{V} \frac{1}{|y|^{q-2}}+\mathcal{O}\left(\frac{1}{|y|^{q-1}}\right), \\
\nabla G(y, \infty)=\frac{q-2}{V} \frac{1}{|y|^{q-1}} \nabla|y|+\mathcal{O}\left(\frac{1}{|y|^{q}}\right)=\mathcal{O}\left(\frac{1}{|y|^{q-1}}\right) \quad \text { as } y \rightarrow \infty .
\end{gathered}
$$

Remark. For $q=2$ there is a logarithmic singularity at $\infty$,

$$
G(y, \infty)=V-U^{\omega}(y), \quad y \in K^{\infty} .
$$

We now turn to the important special case $K=\bar{\Omega}$ with $\Omega$ as in Section 1.2. If $0 \in \Omega$, the Green function $G(y, \infty)$ for $\Omega^{\infty}$ with pole at $\infty$ will be related to the Green function $G^{\prime}(z, 0)$ for the bounded domain $\Omega^{\prime}=j\left(\Omega^{\infty}\right)$ with pole at 0 by the formula

$$
G(y, \infty)=\frac{1}{|y|^{q-2}} G^{\prime}(j(y), 0), \quad y \in \Omega^{\infty} .
$$

We will need the following results on Green functions in $\mathbb{R}^{q}(q \geq 2)$ :

Proposition 2.2. (i) Let $\Sigma=\partial \Omega$ be of class $C^{1, \alpha}$. Then the interior Green function $G(y, x)$ is of class $C^{1}$ on $\bar{\Omega}-\{x\}$ and the (interior) normal derivative $\partial G(y, x) / \partial n_{y}$ is strictly positive on $\Sigma$. Moreover, there is a constant $c=c(\Omega)$ depending only on $\Omega$ such that, with $d_{x}=d(x, \Sigma)$,

$$
\left|\nabla_{y} G(y, x)\right| \leq \frac{c}{|y-x|^{q-1}}, \quad \forall y \in \bar{\Omega} ; \quad \frac{\partial G}{\partial n_{y}}(y, x) \leq \frac{c}{d_{x}^{q-1}}, \quad \forall y \in \Sigma .
$$

There are corresponding results for exterior Green functions $G(y, x)$ with finite pole $x$, but now $\partial G(y, x) / \partial n_{y} \leq c^{\prime}\left(1+d_{x}\right) / d_{x}^{q-1}$ for $y \in \Sigma$. Similarly, $G(y, \infty)$ is of class $C^{1}$ on $\operatorname{clos} \Omega^{\infty}-\{\infty\}$ and there is a positive constant $m=m(\Sigma)$ such that

$$
\frac{\partial G}{\partial n_{y}}(y, \infty) \geq m, \quad \forall y \in \Sigma .
$$

(ii) In the case of convex $\Omega$ there are positive constants $c$ and $m$ such that (2.8) and (2.9) hold for all y outside an exceptional boundary set of "area measure" zero.

Proof. (i) For the $C^{1, \alpha}$ case the results on the interior Green function $G(y, x)$ go back to Lyapunov [26] and Eydus [5], see Widman [34], [35] who actually considered more general Lyapunov-Dini domains $\Omega$. For the exterior Green functions one may use inversion.

(ii) The convex case. For convex $\Omega \subset \mathbb{R}^{3}$ the Green functions with pole $x$ in $\Omega$ or at $\infty$ have been studied by Gabriel [8]-[10]. He proved that the level surfaces for these functions are strictly convex, and that along the orthogonal trajectories of the interior level surfaces, $|\nabla G(y, x)|$ is strictly decreasing as $y$ moves from the pole towards $\Sigma$. The same method shows that $|\nabla G(y, \infty)|$ is strictly increasing towards 
$\Sigma$ along such trajectories in the exterior, cf. Monterie [27]. One could use this fact to obtain (2.9). Gabriel's proofs readily extend to other dimensions.

(iia) We will prove (2.8) with a constant $c$ depending only on $q$. It is sufficient to consider the case where $y \in \Sigma$ and $\Sigma$ is smooth, otherwise one can work with a level surface $\Sigma_{\delta}:\{G=\delta\}$ and take a limit if necessary, cf. Proposition 4.1 below. In the proof we take $q \geq 3$ and compare $\Omega$ with a half-space; for the case $q=2$ (with $c=2$ ) cf. Nevanlinna [28], section 63. Let $Y$ be the tangent hyperplane to $\Sigma$ at $y$ and let $H$ be the half-space with boundary $Y$ that contains $\Omega$. By the comparison principle, $G(z, x)$ is majorized for $z \in \Omega$ by the Green function

$$
\tilde{G}(z, x)=\frac{1}{|x-z|^{q-2}}-\frac{1}{\left|x^{\prime}-z\right|^{q-2}}
$$

for $H$ with pole at $x ; x^{\prime}$ is the reflection of $x$ in $Y$. Thus

$$
\left|\nabla_{y} G(y, x)\right|=\frac{\partial G}{\partial n_{y}}(y, x) \leq \frac{\partial \tilde{G}}{\partial n_{y}}(y, x)=\frac{2(q-2)(x-y) \cdot n_{y}}{|x-y|^{q}} \leq \frac{2(q-2)}{|x-y|^{q-1}} .
$$

(iib) For (2.9) we use the fact that $\Omega$ satisfies an exterior sphere condition with fixed radius $r$. For each $y \in \Sigma$, we consider a sphere $S_{y}$ with radius $r$ in $\Omega^{\infty}$ which is tangent to $\Sigma$ at $y$, as well as the concentric sphere $S_{y}^{\prime}$ of radius $\frac{1}{2} r$. Letting $\beta>0$ be the minimum of $G=G(\cdot, \infty)$ on the union of the spheres $S_{z}^{\prime}, z \in \Sigma$, we define $F=F_{y}$ as the harmonic function on the "annular" domain $A_{y}$ between $S_{y}$ and $S_{y}^{\prime}$ with boundary values 0 on $S_{y}$ and $\beta$ on $S_{y}^{\prime}$. Since $G \geq F$ on $A_{y}$,

$$
\frac{\partial G}{\partial n_{y}}(y) \geq \frac{\partial F}{\partial n_{y}}(y)
$$

wherever $\partial G / \partial n_{y}$ exists. The right-hand side is equal to a positive constant $m$ which depends only on $\beta, r$ and $q$.

Remark. The uniform exterior sphere condition also implies that $\partial G(y, x) / \partial n_{y} \leq$ $C\left(d_{x}\right)$ for $x \in \Omega$ and all $y \in \Sigma$ where the normal derivative exists. This may be proved by comparison of $G(y, x)$ with a suitable harmonic function on the intersection of $\Omega$ with the annular domain bounded by $S_{y}$ and a concentric sphere of radius $r+\frac{1}{2} d_{x}$.

2.3. Harnack inequalities. We will need some properties of harmonic functions in $\mathbb{R}^{q}$ related to the Poisson integral for a ball, cf. Gilbarg and Trudinger [11], Hayman and Kennedy [12].

Poisson integral. If $W$ is harmonic in the ball $B\left(x_{0}, R\right)$ and (piecewise) continuous on the closed ball, then

$$
W(x)=\frac{1}{R} \int_{S\left(x_{0}, R\right)} \frac{R^{2}-\left|x-x_{0}\right|^{2}}{|x-y|^{q}} W(y) d \sigma(y), \quad x \in B\left(x_{0}, R\right),
$$

where $\sigma$ denotes adjusted area measure as in Section 1.3. Hence, taking the gradient under the integral sign,

$$
\left|\nabla W\left(x_{0}\right)\right| \leq \frac{q}{R} \sup _{S\left(x_{0}, R\right)}|W(y)|
$$


Standard Harnack inequalities. If $T$ is harmonic and $\geq 0$ in the ball $B\left(x_{0}, R\right)$, then for $\left|x-x_{0}\right|=r<R$,

$$
\frac{(R-r) R^{q-2}}{(R+r)^{q-1}} T\left(x_{0}\right) \leq T(x) \leq \frac{(R+r) R^{q-2}}{(R-r)^{q-1}} T\left(x_{0}\right) .
$$

By inversion, if $T$ is harmonic on the exterior $R^{\prime}<\left|x-x_{0}\right| \leq \infty$ of $B\left(x_{0}, R^{\prime}\right)$, then for $\left|x-x_{0}\right|=R^{\prime \prime}>R^{\prime}$,

$$
T(x) \leq \frac{R^{\prime \prime}+R^{\prime}}{\left(R^{\prime \prime}-R^{\prime}\right)^{q-1}} L(T), \quad \text { where } L(T)=\lim _{x \rightarrow \infty}|x|^{q-2} T(x) .
$$

For arbitrary bounded domains $\Omega$ containing 0 or unbounded domains $\Omega^{\infty}$ containing $\infty$, with boundary $\Sigma$, one may use (2.12), (2.13) and a covering argument for the subset $E$ of those points $x$ for which $d_{x}=d(x, \Sigma) \geq \delta>0$ to obtain

General Harnack inequalities. For nonnegative harmonic functions $T$,

$$
T(x) \leq c_{1}\left(d_{x}\right) T(0), \quad \forall x \in \Omega ; \quad T(x) \leq c_{2}\left(d_{x}\right) L(T), \forall x \in \Omega^{\infty} .
$$

We need the following more precise estimates for special domains:

Proposition 2.3. Let $\Sigma=\partial \Omega \subset \mathbb{R}^{q}$ be of class $C^{1, \alpha}$, or suppose that $\Omega$, or $\Omega^{\infty}$, respectively, satisfies a uniform interior sphere condition. Then there are a constant $c_{0}($ if $0 \in \Omega)$ and a constant $c_{\infty}$ such that for all nonnegative harmonic functions $T$ on $\Omega$, and on $\Omega^{\infty}$, respectively,

$$
T(x) \leq \frac{c_{0}}{d_{x}^{q-1}} T(0), \quad \forall x \in \Omega ; \quad T(x) \leq \frac{c_{\infty}\left(1+d_{x}\right)}{d_{x}^{q-1}} L(T), \quad \forall x \in \Omega^{\infty} .
$$

The second estimate will hold in particular on the exterior of a compact convex set.

Proof. (i) The $C^{1, \alpha}$ case. Let $T$ be nonnegative harmonic on a Lipschitz domain $\Omega \ni 0$. By the work of Hunt and Wheeden [13], cf. Jerison and Kenig [14], there is then a finite positive measure $\mu$ on $\Sigma$ such that

$$
T(x)=\int_{\Sigma} K(y, x) d \mu(y),
$$

where the kernel $K(y, x)$ is equal to the Radon-Nikodym derivative $d \omega_{x}(y) / d \omega_{0}(y)$. Now in the $C^{1, \alpha}$ case

$$
K(y, x)=\frac{\partial G(y, x) / \partial n_{y}}{\partial G(y, 0) / \partial n_{y}}
$$

hence by Proposition 2.2, setting $\min _{\Sigma} \partial G(y, 0) / \partial n_{y}=m_{0}$,

$$
T(x) \leq \frac{c}{d_{x}^{q-1}} \frac{1}{m_{0}} \int_{\Sigma} d \mu(y)=\frac{c}{m_{0} d_{x}^{q-1}} T(0), \quad \forall x \in \Omega .
$$

(ii) The sphere condition case. Suppose $\Omega \ni 0$ satisfies the interior sphere condition with radius $R$. We may assume that $R<d(0, \Sigma)$. There will then be a constant $M$ such that for the points $z \in \Omega$ with $d_{z} \geq R, T(z) \leq M T(0)$. For a point $x$ with $d_{x}<R$ there must be a unique nearest point $y \in \Sigma$; furthermore, the sphere $S(z, R)$ through $y$ with radius $R$ and center $z$ on the extended directed segment $[y, x]$ must belong to $\bar{\Omega}$. Now $(2.12)$ shows that $T(x) \leq\left(2 R / d_{x}\right)^{q-1} T(z)$.

The second inequality (2.15) follows from the first by inversion. 
2.4. Capacity regularity. For nonempty compact $K \subset \mathbb{R}^{q}(q \geq 2)$ we let $K_{\rho}$ be the closed $\rho$-neighborhood of $K(\rho>0)$.

Definition 2.4. $K$ (or its interior) will be called capacity-regular if there are positive constants $A$ and $B$ such that

$$
\operatorname{cap} K_{\rho} \leq \operatorname{cap} K+A \rho, \quad \text { or } \quad V\left(K_{\rho}\right) \geq V(K)-B \rho, \quad \forall \rho>0 .
$$

Since cap $K_{\rho}=\mathcal{O}(\rho)$ for $\rho \rightarrow \infty$, condition (2.16) is a constraint only for $\rho \rightarrow 0$.

Proposition 2.5. $C^{1, \alpha}$ and bounded convex domains $\Omega$ are capacity-regular. Lipschitz domains $\Omega$ are capacity-regular at least for $q \geq 3$.

Proof. For our main results in dimension $q \geq 2$ we only need the case of $C^{1, \alpha}$ and convex domains. For those, capacity regularity is easy to prove. Set $\bar{\Omega}=K$. In the $C^{1, \alpha}$ case, $K_{\rho}$ is enclosed by a level surface $\Sigma_{\delta}:\{G(y, \infty)=\delta\}$ with $\delta=C \rho$, hence $V\left(K_{\rho}\right) \geq V\left(\Sigma_{\delta}\right)=(1-C \rho) V(K)$. In the convex case, one may blow up $K$ by a factor $1+C \rho$ from a suitable center to cover $K_{\rho}$.

Nick Korevaar has shown us how to prove the Lipschitz result $(q \geq 3)$. Define

$$
C(K)=\inf \int_{\mathbb{R}^{q}}|\nabla u|^{2}, \quad K=\bar{\Omega},
$$

where $u$ runs over all functions in $C_{0}^{\infty}$ which are $\geq 1$ on $K$; the Robin constant $V(K)$ equals const $/ C(K)$, cf. Adams and Hedberg [1] or Gilbarg and Trudinger [11] (1983 edition, p. 27). Thus it will be sufficient to compare $C\left(K_{\rho}\right)$ with $C(K)$.

The idea of the proof is to construct a diffeomorphism $y=\Psi(x)$ of $\mathbb{R}^{q}$ which maps $K$ onto a neighborhood of $K_{\rho}$ and which is equal to the identity plus $\mathcal{O}(\rho)$. With such a map in hand one can transform "good" functions $u(x)$ for $C(K)$ in (2.17) into good functions $U(y)=u(x)$ for $K_{\rho}$ :

$$
U \in C_{0}^{\infty}, U \geq 1 \text { on } K_{\rho}, \nabla_{y} U(y)=\{1+\mathcal{O}(\rho)\} \nabla_{x} u(x) .
$$

Since also $d y=\{1+\mathcal{O}(\rho)\} d x$ one will conclude that

$$
C\left(K_{\rho}\right) \leq \int\left|\nabla_{y} U(y)\right|^{2} d y \leq\{1+\mathcal{O}(\rho)\} \int\left|\nabla_{x} u(x)\right|^{2} d x \quad \text { as } \quad \rho \rightarrow 0 .
$$

As a first step towards a suitable diffeomorphism we construct a smooth vector field $v$ on $\mathbb{R}^{q}$ which is transverse to $\Sigma$ and has compact support. Consider the points $y \in \Sigma=\partial K$ where there is a well-defined unit outer normal $n_{y}$. (The normals exist almost everywhere relative to area measure.) There will be constants $\delta>0$ and $2 r>0$ (depending on the Lipschitz character of $\Sigma$ ) as follows: for all our points $y$, there is a ball $B(y, 2 r)$ such that the normals to $\Sigma$ at the points $z \in \Sigma \cap B(y, 2 r)$ satisfy $n_{z} \cdot n_{y}>\delta$. We now cover $\Sigma$ by a finite number of the smaller balls $B(y, r)$ and define a piecewise constant vector field $w$ by setting $w(x)=\Sigma_{y} n_{y}$, where the summation is over all $y$ such that $B(y, r) \ni x$. Then $w \cdot n>\delta$ on $\Sigma$ and $w=0$ at distance $\geq r$ from $\Sigma$. The desired smooth vector field $v$ is obtained by convolution with an approximate identity of small support: $v=w \star \phi$. Here we take supp $\phi$ so small that $v \cdot n>\frac{1}{2} \delta$ on $\Sigma$.

One finally defines the diffeomorphism $\Psi$ by flow with the field $v$ over a distance $(3 / \delta) \rho$, so that $\Psi(x)=x+(3 / \delta) \rho v(x)$. For small $\rho$, the set $K_{\rho}$ will then be contained in $\Psi(K)$.

There are compact sets $K$ which fail to be capacity-regular: 
Examples 2.6. Line segments $L$ in $\mathbb{R}^{2}$ or $\mathbb{R}^{3}$ are not capacity-regular. This may be shown by comparing $L_{\rho}$ with rectangles or ellipsoids. Taking for $L$ the segment $[-1,1]$ of the $x_{1}$-axis in $\mathbb{R}^{3}$ one may use inscribed and circumscribed ellipsoids

$$
E: \frac{x_{1}^{2}}{a^{2}}+\frac{x_{2}^{2}+x_{3}^{2}}{b^{2}} \leq 1, \quad \operatorname{cap} E=\frac{2 c}{\pi \log \{(a+c) /(a-c)\}}
$$

(where $c=\sqrt{a^{2}-b^{2}}, a>b>0$ ), cf. Landkof [25], to derive that

$$
\operatorname{cap} L_{\rho} \sim \frac{1}{\pi \log (1 / \rho)} \quad \text { as } \rho \rightarrow 0 .
$$

In $\mathbb{R}^{3}$ a disc would be a good candidate for an example with positive capacity. However, it is easier to treat the union $K$ of the closed unit ball $\bar{B}=\bar{B}(0,1)$ and a segment of the $x_{1}$-axis such as $L=[1,3]$. Here one may use a combination of the equilibrium measures for $\bar{B}_{\rho}$ and $L_{\rho}^{\prime}$, where $L^{\prime}=[2,3]$, to show that

$$
\operatorname{cap} K_{\rho}>\operatorname{cap} K+\frac{c}{\log (1 / \rho)} \quad \text { as } \rho \rightarrow 0, \quad \text { with } c>0 \text {. }
$$

\section{The energy method. Exterior estimate for Fekete potentials}

In this chapter $K$ is an arbitrary compact set in $\mathbb{R}^{q}(q \geq 2)$ of positive capacity, cap $K$, or finite Robin constant $V=V(K)$. As before, $K_{\rho}$ stands for the closed $\rho$ neighborhood of $K$ and $K^{\infty}$ denotes the unbounded component of the complement $K^{c}$ of $K$, including the point at infinity. As in Chapter $1, \omega_{N}=\omega_{N}(K)$ and $\omega=\omega(K)$ denote an (arbitrary) $N$ th order Fekete measure for $K$, and the classical equilibrium measure, respectively. The corresponding potentials are $U^{\omega_{N}}$ and $U^{\omega}$. The energy $I(\omega)$ is equal to the Robin constant $V=V(K)$. For $\omega_{N}$ we use the discrete or Fekete energy, $I^{*}\left(\omega_{N}\right)$.

Our first goal is to obtain a good lower bound for the difference $U^{\omega_{N}}-U^{\omega}$. Here it is convenient to introduce a number $\delta\left(\omega_{N}\right)$ by setting

$$
-\delta\left(\omega_{N}\right) \stackrel{\text { def }}{=} \inf _{x \in K} U^{\omega_{N}}(x)-V .
$$

Since $U^{\omega} \leq V$, Fubini's theorem shows that

$$
-\delta\left(\omega_{N}\right) \leq \int_{K} U^{\omega_{N}} d \omega-V=\int_{K} U^{\omega} d \omega_{N}-V \leq 0 .
$$

(As a rule $\delta\left(\omega_{N}\right)>0$. For example, if $K$ is the boundary $\Sigma$ of a Lipschitz domain $\Omega$ one has $\operatorname{supp} \omega=\Sigma$. It then follows from (3.2) that $U^{\omega_{N}}-V$, which is positive around the Fekete points, must be negative somewhere on $\Sigma$.) It is easy to prove

Proposition 3.1. For all $x \in \mathbb{R}^{q}$,

$$
U^{\omega_{N}}(x)-U^{\omega}(x) \geq-\delta\left(\omega_{N}\right) .
$$

Proof. For $x \in K,(3.3)$ follows from (3.1) and the inequality $U^{\omega}(x) \leq V$. Next observe that $U^{\omega_{N}}-U^{\omega}$ is harmonic on the complement $K^{c}$ of $K$, including the point at $\infty$. Now let $x \in K^{c}$ tend to a point $x_{0} \in K$. Then since $U^{\omega_{N}}$ is continuous as a function to $\mathbb{R} \cup\{\infty\}$,

$$
\liminf _{x \rightarrow x_{0}}\left\{U^{\omega_{N}}(x)-U^{\omega}(x)\right\} \geq U^{\omega_{N}}\left(x_{0}\right)-V \geq-\delta\left(\omega_{N}\right) .
$$

Thus by the minimum principle for harmonic functions, (3.3) will also hold on every component of $K^{c}$. 
3.1. Comparing Fekete potential and energy on $K$. The minimum property of the Fekete $N$-tuples may be used to compare $U^{\omega_{N}}$ on $K$ with $I^{*}\left(\omega_{N}\right)$, cf. Sjögren $[32]:$

Proposition 3.2. One has

$$
\left(1-\frac{1}{N}\right) U^{\omega_{N}}(x) \geq I^{*}\left(\omega_{N}\right), \quad \forall x \in K .
$$

Proof. For $x \in K$ and each $j$, by the minimum property mentioned above,

$$
U_{j}(x) \stackrel{\text { def }}{=} \frac{1}{N-1} \sum_{k=1, k \neq j}^{N} \Phi\left(\left|x-f_{N k}\right|\right) \geq U_{j}\left(f_{N j}\right)=\frac{1}{N-1} \sum_{k=1, k \neq j}^{N} \Phi\left(\left|f_{N j}-f_{N k}\right|\right) .
$$

Averaging over $j=1, \ldots, N$ one finds that

$$
\frac{1}{N} \sum_{j=1}^{N} U_{j}(x)=U^{\omega_{N}}(x) \geq \frac{1}{N} \sum_{j=1}^{N} U_{j}\left(f_{N j}\right)=\frac{N}{N-1} I^{*}\left(\omega_{N}\right) .
$$

3.2. Lower bound for the discrete energy. The basic ingredients used in the derivation below may be found in the classical work of Pólya and Szegö [29].

Proposition 3.3. For every $\omega_{N}$ and all $\rho>0$,

$$
I^{*}\left(\omega_{N}\right)>V\left(K_{\rho}\right)-\frac{1}{N} \Phi(\rho)
$$

Proof. Let $\omega_{N}^{\rho}$ be the probability measure on $K_{\rho}$, obtained from $\omega_{N}$ on $K$ by distributing the charges $1 / N$ at the Fekete points $f_{N k}$ uniformly over the spheres $S\left(f_{N k}, \rho\right)$ of radius $\rho$, centered at those points. The potential $U_{N k}$ of the resulting measure $\omega_{N k}^{\rho}$ on $S\left(f_{N k}, \rho\right)$ satisfies the relations

$$
U_{N k}(x)=\frac{1}{N} \min \left\{\Phi(\rho), \Phi\left(\left|x-f_{N k}\right|\right)\right\} \leq \frac{1}{N} \Phi\left(\left|x-f_{N k}\right|\right) .
$$

Thus for $j \neq k$,

$$
\int U_{N j} d \omega_{N k}^{\rho} \leq \frac{1}{N} \int \Phi\left(\left|x-f_{N j}\right|\right) d \omega_{N k}^{\rho}(x)=\frac{1}{N} U_{N k}\left(f_{N j}\right) \leq \frac{1}{N^{2}} \Phi\left(\left|f_{N j}-f_{N k}\right|\right),
$$

while for $j=k$,

$$
\int U_{N k} d \omega_{N k}^{\rho}=\frac{1}{N^{2}} \Phi(\rho)
$$

Adding up all the terms $\int U_{N j} d \omega_{N k}^{\rho}$, it follows that

$$
I\left(\omega_{N}^{\rho}\right) \leq \frac{1}{N^{2}} \sum_{j, k=1, j \neq k}^{N} \Phi\left(\left|f_{N j}-f_{N k}\right|\right)+\frac{1}{N} \Phi(\rho)=I^{*}\left(\omega_{N}\right)+\frac{1}{N} \Phi(\rho) .
$$

The proof of (3.7) is completed by the observation that the energy $I\left(\omega_{N}^{\rho}\right)$ will be larger than the minimum $V\left(K_{\rho}\right)$ of $I(\mu)$ for probability measures $\mu$ on $K_{\rho}$.

Combining Propositions 3.2 and 3.3 with the definition of $\delta\left(\omega_{N}\right)$ in (3.1) one obtains 
Proposition 3.4. For every $\omega_{N}$ and all $\rho>0$,

$$
-\delta\left(\omega_{N}\right)>\frac{N}{N-1} V\left(K_{\rho}\right)-V(K)-\frac{1}{N-1} \Phi(\rho) .
$$

\subsection{First lower bound for $U^{\omega_{N}}-U^{\omega}$ when $K$ is capacity-regular.}

Theorem 3.5. Let $K \in \mathbb{R}^{q}$ be capacity-regular with constant $B=B(K)$ as in (2.16). Then for $q \geq 3$ there is a constant $c_{1}$ depending only on $B$ and $q$ such that for every $N$ th order Fekete measure $\omega_{N}$ on $K$,

$$
\delta\left(\omega_{N}\right) \leq \frac{c_{1}}{N^{1 /(q-1)}}, \text { hence } U^{\omega_{N}}(x)-U^{\omega}(x) \geq-\frac{c_{1}}{N^{1 /(q-1)}}, \quad \forall x \in \mathbb{R}^{q} .
$$

Proof. By (3.8), (2.16) and (1.2),

$$
-\delta\left(\omega_{N}\right)>\frac{N}{N-1}\{V(K)-B \rho\}-V(K)-\frac{1}{N-1} \frac{1}{\rho^{q-2}}, \quad \forall \rho>0 .
$$

The first inequality in (3.9) now follows by maximization of the right-hand side of (3.10) as a function of $\rho$. Next use Proposition 3.1.

Remark 3.6. For $q=2$ one has $\Phi(\rho)=\log (1 / \rho)$ and then the inequality for $\delta\left(\omega_{N}\right)$ becomes

$$
\delta\left(\omega_{N}\right) \leq c_{1} \frac{\log N}{N} .
$$

3.4. The auxiliary potential $T$. For $q \geq 3$, we now introduce an auxiliary potential $T$ as in Section 1.3:

$$
T=U^{\rho_{N}}=U^{\omega_{N}}-\left(1-\frac{1}{V} \delta\left(\omega_{N}\right)\right) U^{\omega}, \quad \rho_{N}=\omega_{N}-\left(1-\frac{1}{V} \delta\left(\omega_{N}\right)\right) \omega .
$$

Proposition 3.7. The function $T$ is nonnegative throughout $\mathbb{R}^{q}$. It is harmonic on the complement $K^{c}$ of $K$ (including $\infty$ ) and

$$
L(T)=\lim _{x \rightarrow \infty}|x|^{q-2} T(x)=\frac{1}{V} \delta\left(\omega_{N}\right) .
$$

Proof. If $\delta\left(\omega_{N}\right) \geq V$, then $T \geq U^{\omega_{N}}>0$, hence in order to prove $T \geq 0$ we may assume $\delta\left(\omega_{N}\right)<V$. The nonnegativity of $T$ in $\mathbb{R}^{q}$ then follows from the fact that

$$
T \geq U^{\omega_{N}}-\left(1-\frac{1}{V} \delta\left(\omega_{N}\right)\right) V=U^{\omega_{N}}-V+\delta\left(\omega_{N}\right) \geq 0 \text { on } K,
$$

cf. the definition of $\delta\left(\omega_{N}\right)$ in (3.1) and the proof of Proposition 3.1.

For probability measures $\mu$ on $K$, the product $|x|^{q-2} U^{\mu}(x)$ tends to 1 as $x \rightarrow \infty$, hence $T$ satisfies the limit relation (3.13).

3.5. Upper bound for $U^{\omega_{N}}-U^{\omega}$ on the exterior domain $K^{\infty}$. New lower bound.

Theorem 3.8. Let $K \subset \mathbb{R}^{q}, q \geq 3$, be capacity-regular as in Definition 2.4. Then there is a function $c\left(d_{x}\right)$ depending only on $(K$ and $) d_{x}=d(x, K)$ such that for every $N$ th order Fekete measure $\omega_{N}$ on $K$ and every $x \in K^{\infty}$,

$$
-c_{1} \min \left(1, \frac{1}{V d_{x}^{q-2}}\right) \frac{1}{N^{1 /(q-1)}} \leq U^{\omega_{N}}(x)-U^{\omega}(x) \leq T(x) \leq \frac{c\left(d_{x}\right)}{N^{1 /(q-1)}} .
$$

Here $c_{1}$ is the constant from (3.9). 
Proof. Knowing that $T(3.12)$ is harmonic and nonnegative throughout $K^{\infty}$, we can use the second Harnack inequality (2.14) to conclude that for every $\omega_{N}$,

$$
T(x) \leq c_{2}\left(d_{x}\right) L(T)=c_{2}\left(d_{x}\right) \frac{1}{V} \delta\left(\omega_{N}\right), \quad x \in K^{\infty} .
$$

Here $\delta\left(\omega_{N}\right)$ is as in (3.1), cf. Proposition 3.7. The proof of (3.15) is completed with the aid of (3.12) and (3.9):

$$
\begin{gathered}
U^{\omega_{N}}(x)-U^{\omega}(x)=T(x)-\frac{1}{V} \delta\left(\omega_{N}\right) U^{\omega}(x) \leq T(x) \leq \frac{c^{\prime}\left(d_{x}\right)}{N^{1 /(q-1)}} \\
U^{\omega_{N}}(x)-U^{\omega}(x) \geq-\frac{1}{V} \delta\left(\omega_{N}\right) U^{\omega}(x) \geq-\frac{1}{V} \delta\left(\omega_{N}\right) \frac{1}{d_{x}^{q-2}} \geq-\frac{c_{1}}{V} \frac{1}{d_{x}^{q-2}} \frac{1}{N^{1 /(q-1)}} .
\end{gathered}
$$

Refinement 3.9. If $K$ has $C^{1, \alpha}$ outer boundary $\Sigma$ or if $K$ is the closure of a convex domain, Proposition 2.3, (3.13) and (3.9) imply that one may take

$$
c\left(d_{x}\right)=\frac{c^{*}\left(1+d_{x}\right)}{d_{x}^{q-1}}
$$

in (3.15), where $c^{*}$ depends only on $K$.

Remark 3.10. For dimension $q=2$ one may define a nonnegative auxiliary function $T$ by setting $T=U^{\omega_{N}}-U^{\omega}+\delta\left(\omega_{N}\right)$. In this case $L(T)=\delta\left(\omega_{N}\right)$ so that by (3.11) for capacity-regular $K$,

$$
U^{\omega_{N}}(x)-U^{\omega}(x) \leq c^{\prime}\left(d_{x}\right) \frac{\log N}{N}, \quad x \in K^{\infty} .
$$

Remark 3.11. One has $I^{*}\left(\omega_{N}\right)<(1-1 / N) I(\omega)$. Indeed, the harmonic function $u_{j}=U_{j}-U^{\omega}$ on $K^{\infty}$ has $\lim _{x \rightarrow \infty}|x|^{q-2} u_{j}(x)=0$, cf. (3.5), hence by the minimum principle, $\liminf \operatorname{in}_{x \rightarrow \partial_{0} K} u_{j}(x)<0$. It follows that $U_{j}\left(f_{N j}\right)<\sup U^{\omega}=V$. Finally, by $(3.6), I^{*}\left(\omega_{N}\right)<(1-1 / N) V$.

In combination with (3.7) for suitable $\rho=\rho_{N} \rightarrow 0$, it follows that $I^{*}\left(\omega_{N}\right) \rightarrow$ $V=I(\omega)$ as $N \rightarrow \infty$ for every compact set $K$ of positive capacity.

\section{IntEgRAL REPRESENTATIONS FOR POTENTIALS INVOLVING GREEN FUNCTIONS}

Throughout this chapter $\Omega$ is a (bounded) Lipschitz domain in $\mathbb{R}^{q}, q \geq 2$, with boundary $\Sigma$ and exterior domain $\Omega^{\infty}$, cf. Section 1.3. As before, $\sigma$ denotes adjusted area measure $\sigma(y)=\lambda(y) /(q-2) \lambda(S)$ on $\Sigma$ (leaving out $(q-2)$ if $q=2$ ).

4.1. Representation for harmonic functions and potentials on $\Omega$. The following proposition summarizes an important result of Dahlberg [2] concerning harmonic measure on Lipschitz domains, cf. Jerison and Kenig [14] and Kenig [16].

Proposition 4.1. For $x \in \Omega$ let $G(\cdot)=G(\cdot, x)$ be the Green function for $\Omega$ with pole at $x$. There will be an exceptional subset $F=F_{x}$ of $\Sigma=\partial \Omega$ of area measure $\sigma(F)=0$ as follows. Whenever $y \in \Sigma-F$, the inward unit normal $n_{y}$ exists and the normal derivative

$$
\frac{\partial G}{\partial n_{y}}(y)=\lim _{\varepsilon \downarrow 0} \frac{1}{\varepsilon} G\left(y+\varepsilon n_{y}\right)
$$


exists as a finite positive number. As a function of $y$, the derivative is integrable over $\Sigma$. For measurable subsets $E \subset \Sigma$, the harmonic measure

$$
\omega_{x}(E) \text { equals } \int_{E} \frac{\partial G}{\partial n_{y}}(y, x) d \sigma(y) .
$$

Not only is $\omega_{x}$ absolutely continuous with respect to $\sigma$, but the converse is also true.

By the definition of harmonic measure, Proposition 4.1 implies a Green-type representation for functions $u$ of class $C H(\Omega)$ : harmonic functions on $\Omega$ which are continuous up to the boundary. We extend the formula to potentials of measures on the boundary by a limit process.

Theorem 4.2. For Lipschitz $\Omega$ with boundary $\Sigma$ the Green-type representation

$$
u(x)=\int_{\Sigma} u(y) d \omega_{x}(y)=\int_{\Sigma} u(y) \frac{\partial G}{\partial n_{y}}(y, x) d \sigma(y), \quad x \in \Omega,
$$

is valid not only for the functions $u$ in $C H(\Omega)$, but also for the potentials $u=U^{\rho}$ of arbitrary complex measures $\rho$ with support in $\Sigma$ (or with bounded support in $\left.\operatorname{clos} \Omega^{\infty}\right)$.

Proof. (i) We first prove (4.3) for the potential due to a point mass 1 at a point $z_{0} \in \Sigma$. The desired formula is

$$
u_{0}(x) \stackrel{\text { def }}{=} \Phi\left(\left|x-z_{0}\right|\right)=\int_{\Sigma} \Phi\left(\left|y-z_{0}\right|\right) d \omega_{x}(y), \quad x \in \Omega .
$$

Since $\Sigma$ is Lipschitz we can choose a truncated convex open cone $A \subset \Omega^{\infty}$ with vertex at $z_{0}$. Let points $z_{\varepsilon}$ tend to $z_{0}$ along a ray in $A$ as $\varepsilon \downarrow 0$. Then $u_{\varepsilon}(x)=$ $\Phi\left(\left|x-z_{\varepsilon}\right|\right)$ belongs to $C H(\Omega)$, hence (4.3) holds for $u_{\varepsilon}$. Now $u_{\varepsilon}(y) \rightarrow u_{0}(y)$ pointwise for $y \in \Sigma$ as $\varepsilon \downarrow 0$ and $u_{\varepsilon}(y)>0$. (Scaling $\Omega$ to diameter $\leq \frac{1}{2}$, this may also be assumed in the 2-dimensional case.) Hence since

$$
\int_{\Sigma} u_{\varepsilon}(y) d \omega_{x}(y)=u_{\varepsilon}(x) \rightarrow u_{0}(x)
$$

Fatou's lemma shows that $\int u_{0} d \omega_{x}$ exists and is $\leq u_{0}(x)$.

To prove equality one may now use dominated convergence. Indeed, there will be a constant $c>0$ such that $\left|y-z_{\varepsilon}\right| \geq c\left|y-z_{0}\right|$ for all $y \in \Sigma$ and all $z_{\varepsilon}$, hence $u_{\varepsilon}$ will be majorized on $\Sigma$ by a constant multiple of $u_{0}$. (This applies to dimension $q \geq 3$; in dimension 2 there is a majorant $u_{0}+C$.)

(ii) For the potential $u=U^{\rho}$ of an arbitrary complex measure $\rho$ on $\Sigma$, formula (4.3) follows from (4.4) with the aid of Fubini's theorem.

The same proof works if $\operatorname{supp} \rho$ is a bounded subset of $\operatorname{clos} \Omega^{\infty}$.

4.2. Representation at infinity for harmonic functions. We need an extension of the representation in Theorem 4.2 to the exterior domain $\Omega^{\infty}$, in particular for the limit $L(u)=\lim |x|^{q-2} u(x)$ at $\infty$. The proof will use the Kelvin transformation, but this presents a small difficulty: approach to the boundary along straight normals as in (4.1) is not preserved under inversion. However, in (4.1) one may approach $\Sigma$ along any curve tangential to the normal:

Lemma 4.3. Let $\Omega, x, G, F$ and $y \in \Sigma-F$ be as in Proposition 4.1 and let $\gamma$ be a smooth arc in $\Omega$ with end-point $y$ and limit direction along $n_{y}$. For small $\varepsilon>0$, 
let $w_{\varepsilon}$ be the point of $\gamma$ at distance $\varepsilon$ from $y$. Then

$$
\frac{\partial G}{\partial s}(y) \stackrel{\text { def }}{=} \lim _{\varepsilon \downarrow 0} \frac{1}{\varepsilon} G\left(w_{\varepsilon}\right) \quad \text { exists and }=\frac{\partial G}{\partial n_{y}}(y) .
$$

Proof. Since $\Sigma$ is Lipschitz and has a tangent plane at $y$, the set $\Omega-\{x\}$ contains a truncated open convex cone $H$ with vertex $y$ and axis along $n_{y}$. For small $\varepsilon$, both $y_{\varepsilon}=y+\varepsilon n_{y}$ and $w_{\varepsilon}$ belong to $H$. Because the Green function $G$ is positive harmonic on $H$, one may use the Harnack inequalities (2.14) on the maximal ball $B\left(y_{\varepsilon}, R_{\varepsilon}\right)$ around $y_{\varepsilon}$ in $H$ to compare $G\left(w_{\varepsilon}\right)$ with $G\left(y_{\varepsilon}\right)$. The result is

$$
G\left(w_{\varepsilon}\right)=G\left(y_{\varepsilon}\right)\left\{1+\mathcal{O}\left(\frac{\left|w_{\varepsilon}-y_{\varepsilon}\right|}{R_{\varepsilon}}\right)\right\} \quad \text { as } \varepsilon \downarrow 0 .
$$

Thus since $R_{\varepsilon}$ is comparable to $\varepsilon$ while $w_{\varepsilon}-y_{\varepsilon}=o(\varepsilon)$,

$$
\lim \frac{1}{\varepsilon} G\left(w_{\varepsilon}\right)=\lim \frac{1}{\varepsilon} G\left(y_{\varepsilon}\right) .
$$

Proposition 4.4. For Lipschitz $\Omega \subset \mathbb{R}^{q}$ let $G(\cdot)=G(\cdot, x)$ be the Green function for the exterior $\Omega^{\infty}$ with pole at $x$, where $x$ may be $\infty$. Then the outward unit normal $n_{y}$ and the corresponding normal derivative $\partial G / \partial n_{y}$ exist for all $y \in \Sigma=$ $\partial \Omega$ outside an exceptional set $F=F_{x}$ of area measure zero. For any function $u \in C H\left(\Omega^{\infty}\right)$ one has the representations

$$
\begin{gathered}
u(x)=\int_{\Sigma} u(y) d \omega_{x}(y)=\int_{\Sigma} u(y) \frac{\partial G}{\partial n_{y}}(y, x) d \sigma(y), \quad x \in \Omega^{\infty}-\{\infty\}, \\
L(u)=\lim _{x \rightarrow \infty}|x|^{q-2} u(x)=\int_{\Sigma} u(y) d \nu(y) \quad \text { with } d \nu(y)=\frac{\partial G}{\partial n_{y}}(y, \infty) d \sigma(y) .
\end{gathered}
$$

Proof. We limit ourselves to the case $x=\infty$ and the proof of (4.7). For sufficiently smooth $\Sigma$ and $u$, that formula follows immediately from Green's theorem for the domain between $\Sigma$ and the sphere $S(0, R)$ by letting $R \rightarrow \infty$, cf. the asymptotics (2.6).

To deal with general $\Sigma$ and $u$ we take $0 \in \Omega$ so that $|y|^{q-2} G(y)=G^{\prime}(j(y))$ for $y \in \Omega^{\infty}$, where $G^{\prime}(z)$ is the Green function for the reflected domain $\Omega^{\prime}=j\left(\Omega^{\infty}\right)$ with pole at $0(2.7)$. Observe that $\Sigma^{\prime}=\partial \Omega^{\prime}$ is also Lipschitz. Let $F^{\prime}=F_{0}$ be an exceptional subset of $\Sigma^{\prime}$ of the kind described in Proposition 4.1 and define $F=j\left(F^{\prime}\right)$. At $y \in \Sigma-F$ there will then be an outward unit normal $n_{y}$ since there is an inward unit normal $n_{z}$ to $\Sigma^{\prime}$ at $z=j(y)$. Set $y_{\varepsilon}=y+\varepsilon n_{y}$ and $w_{\varepsilon}=j\left(y_{\varepsilon}\right)$. It will be shown that

$$
\frac{\partial G}{\partial n_{y}}(y)=\lim _{\varepsilon \downarrow 0} \frac{1}{\varepsilon} G\left(y_{\varepsilon}\right) \quad \text { exists and }=\frac{1}{|y|^{q}} \frac{\partial G^{\prime}}{\partial n_{z}}(z) .
$$

To this end, notice that inversion of (an initial segment of) the normal $n_{y}$ at $y$ gives a smooth arc in $\bar{\Omega}^{\prime}$ which starts at $z \in \Sigma^{\prime}-F^{\prime}$ in the direction of $n_{z}$. Hence by (2.7) and Lemma 4.3 for $\Omega^{\prime}$ and $F^{\prime}$,

$$
\frac{1}{\varepsilon}\left|y_{\varepsilon}\right|^{q-2} G\left(y_{\varepsilon}\right)=\frac{1}{\varepsilon} G^{\prime}\left(w_{\varepsilon}\right)=\frac{\left|j\left(y_{\varepsilon}\right)-j(y)\right|}{\left|y_{\varepsilon}-y\right|} \frac{G^{\prime}\left(w_{\varepsilon}\right)}{\left|w_{\varepsilon}-z\right|} \rightarrow \frac{1}{|y|^{2}} \frac{\partial G^{\prime}}{\partial n_{z}}(z) \quad \text { as } \varepsilon \downarrow 0,
$$

cf. Section 2.1 for the linear scaling factor at $y$ under inversion. Thus the limit in (4.8) exists and has the indicated value. 
For the proof of (4.7) we now introduce the Kelvin transform $v=k u$ of $u \in$ $C H\left(\Omega^{\infty}\right)$ which will be in $C H\left(\Omega^{\prime}\right)$. Recall that $v(0)$ equals $L(u)(2.3)$. Applying (4.3) to $v$ on $\Omega^{\prime}$, using (4.8) and the appropriate scaling factor for an area element, one obtains the desired result:

$$
L(u)=v(0)=\int_{\Sigma^{\prime}} v(z) \frac{\partial G^{\prime}}{\partial n_{z}}(z) d \sigma(z)=\int_{\Sigma}|y|^{q-2} u(y)|y|^{q} \frac{\partial G}{\partial n_{y}}(y) \frac{d \sigma(y)}{|y|^{2 q-2}} .
$$

For the representation of $u(x)$ when $x$ is finite one may appeal to (2.4).

\subsection{Representation at infinity for potentials.}

Theorem 4.5. Let $\Omega^{\infty}$ be the exterior of Lipschitz $\Omega \subset \mathbb{R}^{q}$, let $\nu=\nu_{q}$ be the representing measure for limits at $\infty$ described in Proposition 4.4 and let $\rho$ be any complex measure on $\Sigma$ or $\bar{\Omega}$. Then the representations (4.6) and (4.7) are also valid for the potential $u=U^{\rho}$ with but one small restriction: the formula

$$
\int_{\Sigma} U^{\rho} d \nu=\int_{\Sigma} U^{\rho}(y) \frac{\partial G}{\partial n_{y}}(y, \infty) d \sigma(y)=L\left(U^{\rho}\right)=\lim _{x \rightarrow \infty}|x|^{q-2} U^{\rho}(x)=\rho(\bar{\Omega})
$$

requires that $\rho(\bar{\Omega})=0$ when $q=2$.

Proof. We limit ourselves to (4.9).

(i) The case $q \geq 3$. Here it will be sufficient to consider the potentials $u(x)=$ $\Phi\left(\left|x-z_{0}\right|\right)$ of point masses 1 at points $z_{0} \in \bar{\Omega}$; as in the case of Theorem 4.2, Fubini's theorem will do the rest. For $z_{0} \in \Omega$ the function $u(x)$ is in $C H\left(\Omega^{\infty}\right)$ and then (4.9) follows directly from Proposition 4.4. Thus we need only show that

$$
\int_{\Sigma} \Phi\left(\left|y-z_{0}\right|\right) d \nu(y)=\lim _{x \rightarrow \infty}|x|^{q-2} \Phi\left(\left|x-z_{0}\right|\right)=1, \quad \forall z_{0} \in \Sigma .
$$

To prove this, one may use Fatou's lemma and dominated convergence as in the proof of Theorem 4.2, now approaching $z_{0} \in \Sigma$ by points $z_{\varepsilon}$ inside $\Omega$ on a suitable ray.

(ii) Dimension $q=2$ is harder since in this case, potentials of point masses 1 are not harmonic at $\infty$. However, constants are. It will be enough to show that $\int u_{0} d \nu=0$ where $u_{0}(x)=\log \left(1 /\left|x-z_{0}\right|\right)-U^{\omega}(x)$ with arbitrary $z_{0} \in \Sigma$. Setting $v_{\varepsilon}(x)=\log \left(1 /\left|x-z_{\varepsilon}\right|\right)-U^{\omega}(x)+V$ with $z_{\varepsilon} \in \Omega$ approaching $z_{0}$ as in part (i), we may assume $v_{\varepsilon}(y)>0$ for $y \in \Sigma($ scale $\Omega$ to diameter $<1)$. Now $v_{\varepsilon} \in C H\left(\Omega^{\infty}\right)$ so that $\int v_{\varepsilon} d \nu=L\left(v_{\varepsilon}\right)=V$, while $v_{\varepsilon} \rightarrow v_{0}=u_{0}+V$ pointwise on $\Sigma$. Thus by Fatou's lemma $\int v_{0} d \nu$ exists and is $\leq V$. Finally, since $v_{\varepsilon} \leq v_{0}+C$ on $\Sigma$, dominated convergence shows that $\int v_{0} d \nu=V$, hence $\int u_{0} d \nu=0$.

4.4. Representation for the equilibrium measure. As a corollary to Theorem 4.5 we obtain a representation for the equilibrium measure in terms of the normal derivative of the Green function with pole at $\infty$ :

Theorem 4.6. Let $K$ be the closure or the boundary of a Lipschitz domain $\Omega$. Then the equilibrium measure $\omega$ for $K$ on $\Sigma=\partial \Omega$ has the representation

$$
\begin{aligned}
& d \omega(y)=V d \nu(y)=V \frac{\partial G}{\partial n_{y}}(y, \infty) d \sigma(y) \text { if } q \geq 3 \\
& d \omega(y)=\frac{\partial G}{\partial n_{y}}(y, \infty) d \sigma(y) \text { if } q=2
\end{aligned}
$$


Proof. Let $\nu=\nu_{q}$ be the representing measure on $\Sigma$ for the value $\lim _{x \rightarrow \infty}|x|^{q-2} u(x)$ as $u$ runs over the class $C H\left(\Omega^{\infty}\right)$. Let $z$ be any point in $\bar{\Omega}$.

(i) For $q \geq 3$ and $u(x)=\Phi_{q}(|x-z|)$, Theorem 4.5 shows that

$$
U^{\nu}(z)=\int_{\Sigma} \Phi_{q}(|y-z|) d \nu(y)=L(u)=1 .
$$

Now if a positive measure on $\bar{\Omega}$ has constant potential on $\bar{\Omega}$, it must be a multiple of the equilibrium measure. This follows readily from Fubini's theorem; in the present case:

$$
V \nu(\Sigma)=\int_{\Sigma} U^{\omega} d \nu=\int_{\Sigma} U^{\nu} d \omega=1
$$

Thus $V \nu$ is a probability measure on $\Sigma$, and its potential equals $V$ everywhere on $\bar{\Omega}$. However, $\omega$ is the unique probability measure on $\Sigma$ with that property, so that $\omega=V \nu$.

(ii) If $q=2$ one may take $u \equiv 1$ in formula (4.7) to show that $\nu_{2}(\Sigma)=1$. We now set $u(x)=\Phi_{2}(|x-z|)-\Phi_{2}\left(\left|x-z_{0}\right|\right)$ with $z_{0} \in \bar{\Omega}$ fixed. Here formula (4.9) gives $\int_{\Sigma} u d \nu_{2}=L(u)=0$, hence

$$
U^{\nu}(z)=\int_{\Sigma} \Phi_{2}(|y-z|) d \nu_{2}(y)=c(\text { onstant }), \quad z \in \bar{\Omega} .
$$

This time Fubini's theorem shows that $c=\int U^{\nu} d \omega=V$, so that $\nu_{2}=\omega$.

Remark 4.7. An alternative proof of Theorem 4.6 may be obtained as follows. Set $G(y)=G(y, \infty)$ on $\Omega^{\infty}, G(y)=0$ on $\bar{\Omega}$. Then the relation between $G$ and $U^{\omega}$ implies that in the sense of distributions

$$
V \Delta G=-\Delta U^{\omega}=(q-2) \lambda(S) \omega
$$

(this is for dimension $\geq 3$, cf. (2.5)). For sufficiently smooth $\Sigma$, Green's theorem may now be applied to $\langle\Delta G, \phi\rangle=\int G \Delta \phi$ with $\phi \in C_{0}^{\infty}$ to show that

$$
\Delta G=\frac{\partial G}{\partial n} \lambda=(q-2) \lambda(S) \frac{\partial G}{\partial n} \sigma,
$$

where $n$ is the normal pointing into $\Omega^{\infty}$. The argument can be extended to Lipschitz surfaces $\Sigma$ by a limit process, using results on the nontangential maximal function for $\nabla G$ such as may be found in Kenig [16].

\section{Final estimates for Fekete potentials and fields. Smaller fields}

5.1. Estimating Fekete potentials and fields. Let $\Omega \subset \mathbb{R}^{q}$ with boundary $\Sigma$ be convex or of class $C^{1, \alpha}$. In particular $K=\bar{\Omega}$ will then be capacity-regular. As before, the complement of $\bar{\Omega}$ is called $\Omega^{\infty}$ and $d_{x}=d(x, \Sigma)$.

Proof of Theorem 1.1. Here $q \geq 3$.

(i) The lower bound in (1.4) follows from Theorems 3.5 and 3.8.

For the upper bound we make use of the nonnegative auxiliary potential $T$ introduced in (1.7):

$$
T=U^{\omega_{N}}-\left(1-\frac{1}{V} \delta\left(\omega_{N}\right)\right) U^{\omega}=U^{\rho_{N}}, \quad \text { with } \operatorname{supp} \rho_{N} \subset \Sigma .
$$


(ii) For $x \in \Omega^{\infty}$, the upper bound for $U^{\omega_{N}}(x)-U^{\omega}(x)$ in (1.4) follows from Theorem 3.8 in conjunction with Refinement 3.9:

$$
U^{\omega_{N}}(x)-U^{\omega}(x) \leq T(x) \leq \frac{c\left(d_{x}\right)}{N^{1 /(q-1)}}=\frac{c^{*}\left(1+d_{x}\right)}{d_{x}^{q-1}} \frac{1}{N^{1 /(q-1)}} .
$$

(iii) For $x \in \Omega$, the upper bound follows from the inequalities (1.11) for $T(x)$ in conjunction with Proposition 2.2, followed by formula (3.13) for $L(T)$ and inequality $(3.9)$ for $\delta\left(\omega_{N}\right)$ :

$$
U^{\omega_{N}}(x)-U^{\omega}(x) \leq T(x) \leq \frac{c^{\prime \prime}}{d_{x}^{q-1}} L(T)=\frac{c^{\prime \prime}}{d_{x}^{q-1}} \frac{1}{V} \delta\left(\omega_{N}\right) \leq \frac{c^{+}}{d_{x}^{q-1}} \frac{1}{N^{1 /(q-1)}} .
$$

Remark 5.1. For the planar case our method gives

$$
-c_{1} \frac{\log N}{N} \leq U^{\omega_{N}}(x)-U^{\omega}(x) \leq \frac{c_{2}\left(1+d_{x}\right)}{d_{x}} \frac{\log N}{N}, \quad \forall x \notin \Sigma,
$$

cf. Remarks 3.6 and 3.10. We conjecture that the numerator $\log N$ in these inequalities can be replaced by 1, cf. Korevaar's survey [21] for supporting arguments.

Proof of Theorem 1.2. Again, $q \geq 3$. By (1.4),

$$
\left|U^{\omega_{N}}(x)-U^{\omega}(x)\right| \leq c_{2}^{\prime} \frac{1+d_{x}}{d_{x}^{q-1}} \frac{1}{N^{1 /(q-1)}}, \quad x \notin \Sigma .
$$

The desired inequality (1.5) for $\mathcal{E}^{\omega_{N}}-\mathcal{E}^{\omega}$ now follows from (5.5) if one applies the gradient inequality $(2.11)$ to $W=U^{\omega_{N}}-U^{\omega}$ on the balls $B\left(x, \frac{1}{2} d_{x}\right)$.

5.2. Distributions of point charges which produce very small interior fields. In the foregoing we have considered the differences

$$
U^{\mu_{N}}-U^{\omega} \text { and } \mathcal{E}^{\mu_{N}}-\mathcal{E}^{\omega}
$$

for the case where $\mu_{N}$ minimizes the potential energy $I^{*}\left(\mu_{N}\right)$ for distributions of $N$ point charges $1 / N$ on $K$. One may also ask a different question:

Problem 5.2. Let $K \subset \mathbb{R}^{q}$ be a compact set of positive capacity. How small can one make the differences in (5.6) (away from the outer boundary $\partial_{0} K$ ) if one allows arbitrary distributions $\mu_{N}=\mu_{N}\left(\zeta_{1}, \ldots, \zeta_{N}\right)$ of point charges $1 / N$ on $\partial_{0} K$ ?

For the unit circle $C_{1}:\{|z|=1\}$ in the complex plane $\mathbb{C}$, the $N$ th roots of unity form a Fekete $N$-tuple. Here the differences $\left|\mathcal{E}^{\omega_{N}}-\mathcal{E}^{\omega}\right|$ become exponentially small outside a fixed neighborhood of $C_{1}$ as $N \rightarrow \infty$. However, for Jordan curves $\Gamma \subset \mathbb{C}$ different from circles, very small differences (5.6) are associated with charges $1 / N$ at the conformal images of $N$ th roots of unity,

$$
\zeta_{k}=\phi\left(e^{i 2 \pi k / N}\right), \quad k=1, \ldots, N,
$$

rather than Fekete points. Here $\phi$ is a conformal map from the exterior of the unit circle onto the exterior of $\Gamma$ such that $\phi(\infty)=\infty$. For analytic Jordan curves $\Gamma$, the corresponding differences (5.6) are $\mathcal{O}\left(e^{-c N}\right)$ at distance $\geq \delta>0$ from $\Gamma$ as $N \rightarrow \infty$, with $c=c(\delta)>0$, cf. Korevaar and Geveci [22]. Even for arbitrary bounded domains $\Omega$, charges at points (5.7) on the outer boundary $\Gamma=\partial_{0} \Omega$ are good for relatively small fields inside $\Omega$, cf. Korevaar [17].

Problem 5.2 is especially challenging in the case of the unit sphere $S=S(0,1)$ in, say, $\mathbb{R}^{3}$. Preliminary numerical results of Kuijlaars and Voogd in Amsterdam 
suggest that for the sphere $S$, the Fekete fields $\mathcal{E}^{\omega_{N}}$ do not become extremely small on interior balls $B(0, r)(r<1)$ as $N \rightarrow \infty$. Cf. also the behavior of the dipole moments for the least energy states in the table by Erber and Hockney [4] for $3 \leq N \leq 112$. But how small can one make the fields $\mathcal{E}^{\mu_{N}}$ on interior balls if one allows arbitrary carrying $N$-tuples

$$
Z_{N}=\left(\zeta_{1}, \ldots, \zeta_{N}\right)
$$

for the point charges $1 / N$ on $S$ ? The precise order of smallness is not known, but one has

Theorem 5.3. (Korevaar and Meyers [24]). On the unit sphere $S \subset \mathbb{R}^{3}$ there are special $N$-tuples of points $Z_{N}$ such that for the associated distributions $\mu_{N}=$ $\mu_{N}\left(Z_{N}\right)$ and $N \rightarrow \infty$, the differences $(5.6)$ become as small as $\mathcal{O}\left(e^{-c N^{1 / 3}}\right)$ outside any given neighborhood of $S$ (with $c>0$ depending on the neighborhood). However, the differences can not become of smaller order than $e^{-c N^{1 / 2}}$.

The first author has conjectured that the latter order is achieved for the Fekete $N$-tuples $Z_{N}=\left(\zeta_{N 1}, \ldots, \zeta_{N N}\right)$ which correspond to the potential in (1.1) with $\Phi(r)=1 / \sqrt{r^{2}+a^{2}}(a>0$ arbitrary). For a proof by (multidimensional) complex analysis it would be sufficient to show that the points in such $N$-tuples are wellseparated:

$$
\left|\zeta_{N j}-\zeta_{N k}\right| \geq \frac{\delta}{\sqrt{N}}, \quad j \neq k,
$$

for some constant $\delta>0$ independent of $N$, see Korevaar [20].

$N$-tuples $Z_{N}$ for which the associated charge distributions $\mu_{N}$ make the differences (5.6) very small (away from $S$ ) provide good nodes for Chebyshev-type quadrature formulas on $S$, that is, quadrature formulas in which all $N$ nodes carry the same weight, see Korevaar and Meyers [24] and Korevaar [20].

\section{REFERENCES}

1. D.R. Adams and L.I. Hedberg, Function spaces and potential theory, Grundlehren Math. Wiss., vol. 314, Springer-Verlag, Berlin, 1996. MR 97j:46024

2. B.E.J. Dahlberg, Estimates of harmonic measure, Arch. Rational Mech. Anal. 65 (1977), 275-288. MR 57:6470

3. 80c:31003

4. T. Erber and G.M. Hockney, Complex systems: Equilibrium configurations of $N$ equal charges on a sphere $(2 \leq N \leq 112)$, Publication 95-075-T, Fermi National Accelerator Laboratory, 1995.

5. D.M. Eydus, Estimates on the derivatives of Green's function, (Russian), Dokl. Akad. Nauk SSSR 106 (1956), 207-209. MR 17:960

6. M. Fekete, Über die Verteilung der Wurzeln bei gewissen algebraischen Gleichungen mit ganzzahligen Koeffizienten, Math. Z. 17 (1923), 228-249.

7. O. Frostman, Potentiel d'équilibre et capacité des ensembles avec quelques applications à la théorie des fonctions, Dissertation, Lunds Univ. Mat. Sem. 3 (1935), 1-118, Zbl 13-063.

8. R.M. Gabriel, An extended principle of the maximum for harmonic functions in 3 dimensions, J. London Math. Soc. 30 (1955), 388-401. MR 17:358

9. __ A result concerning convex level surfaces of 3-dimensional harmonic functions, J. London Math. Soc. 32 (1957), 286-294. MR 19:848

10. - Further results concerning the level surfaces of the Green's function for a 3-dimensional convex domain (I), (II), J. London Math. Soc. 32 (1957), 295-302, 303-306. MR 19:848. 
11. D. Gilbarg and N.S. Trudinger, Elliptic partial differential equations of second order, Grundlehren Math. Wiss., vol. 224, Springer-Verlag, Berlin, 1983. MR 86c:35035

12. W.K. Hayman and P.B. Kennedy, Subharmonic functions I, London Math. Soc. Monographs, vol. 9, Academic Press, London, 1976. MR 57:665

13. R.A. Hunt and R.L. Wheeden, Positive harmonic functions on Lipschitz domains, Trans. Amer. Math. Soc. 147 (1970), 507-527. MR 43:547

14. D.S. Jerison and C.E. Kenig, Boundary value problems on Lipschitz domains, Studies in partial differential equations (W. Littman, ed.), M.A.A. Studies in Math., vol. 23, 1982, pp. 1-68. MR 85f:35057

15. O.D. Kellogg, Foundations of potential theory, Grundlehren Math. Wiss., vol. 31, SpringerVerlag, Berlin, 1929.

16. C.E. Kenig, Harmonic analysis techniques for second order elliptic boundary value problems, CBMS Reg. Conf. Ser. in Math., vol. 83, Amer. Math. Soc., Providence, R.I, 1994. MR 96a:35040

17. J. Korevaar, Asymptotically neutral distributions of electrons and polynomial approximation, Ann. of Math. 80 (1964), 403-410. MR 29:6031

18. _ Equilibrium distributions of electrons on roundish plane conductors I, II, Indag. Math. 36 (1974), 423-437, 438-456. MR 50:13477a,b

19. , Problems of equilibrium points on the sphere and electrostatic fields, Report 76-03, Dept. of Mathematics, Univ. of Amsterdam, 1976.

20. - Chebyshev-type quadratures: use of complex analysis and potential theory, Complex Potential Theory (P.M. Gauthier and G. Sabidussi, eds.), Kluwer, Dordrecht, 1994, pp. 325364. MR 96g:41029

21. ries, Bolyai Soc. Math. Studies, vol. 5, Budapest, 1996, pp. 35-62. CMP 97:08

22. J. Korevaar and T. Geveci, Fields due to electrons on an analytic curve, SIAM J. Math. Anal. 2 (1971), 445-453. MR 44:2939

23. J. Korevaar and R.A. Kortram, Equilibrium distributions of electrons on smooth plane conductors, Indag. Math. 45 (1983), 203-219. MR 85j:30051.

24. J. Korevaar and J.L.H. Meyers, Spherical Faraday cage for the case of equal point charges and Chebyshev-type quadrature on the sphere, Integral Trans. Spec. Funct. 1 (1993), 105-117. MR 97g:41046

25. N.S. Landkof, Foundations of modern potential theory, Grundlehren Math. Wiss., vol. 180, Springer-Verlag, Berlin, 1972. MR 50:2520

26. M.A. Liapounoff, Sur certaines questions qui se rattachent au problème de Dirichlet, J. Math. Pures Appl. (5) 4 (1898), 241-311.

27. M.A. Monterie, Studies in potential theory, Ph.D. thesis, Free University, Amsterdam, 1995.

28. R. Nevanlinna, Analytic functions, Grundlehren Math. Wiss., vol. 162, Springer-Verlag, Berlin, 1970, English transl. MR 43:5003

29. G. Pólya and G. Szegö, Über den transfiniten Durchmesser (Kapazitätskonstante) von ebenen und räumlichen Punktmengen, J. Reine Angew. Math. 165 (1931), 4-49, Zbl 2-136.

30. Ch. Pommerenke, Polynome und konforme Abbildung, Monatsh. Math. 69 (1965), 58-61. MR 30:4919

31. __ Über die Verteilung der Fekete-Punkte, Math. Ann. 168 (1967), 111-127; II, Math. Ann. 179 (1969), 212-218. MR 34:6057; MR 40:377.

32. P. Sjögren, On the regularity of the distribution of the Fekete points of a compact surface in $\mathbb{R}^{n}$, Ark. Mat. 11 (1973), 147-151. MR 49:5381

33. J. Wermer, Potential theory, Lecture Notes in Math., vol. 408, Springer-Verlag, Berlin, 1974. MR 56:12284

34. K.-O. Widman, On the boundary values of harmonic functions in $\mathbb{R}^{3}$, Ark. Mat. 5 (1964), 221-230. MR 34:1544

35. - Inequalities for the Green function and boundary continuity of the gradient of solutions of elliptic differential equations, Math. Scand. 21 (1967), 17-37. MR 39:621

Department of Mathematics, University of Amsterdam, Plantage Muidergracht 24, 1018 TV Amsterdam, Netherlands

E-mail address, J. Korevaar: korevaar@wins.uva.nl 